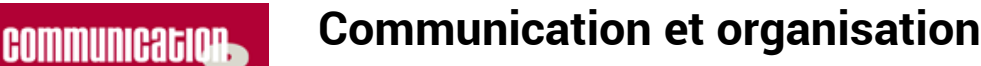

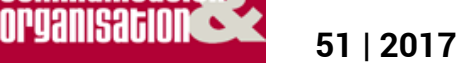

Les nouvelles cultures de l'information dans les organisations

\title{
Le soin-communicant
}

Un espace de médiation en vue de la construction d'une professionnalité soignante

The communicating-care. A mediation space for building a nursing

professionalism

Philippe Viard

\section{OpenEdition}

Journals

Édition électronique

URL : http://journals.openedition.org/communicationorganisation/5578

DOI : 10.4000/communicationorganisation.5578

ISBN : 979-10-300-0146-4

ISSN : 1775-3546

Éditeur

Presses universitaires de Bordeaux

Édition imprimée

Date de publication : 1 juin 2017

Pagination : 141-156

ISBN : 979-10-300-0162-4

ISSN : 1168-5549

Référence électronique

Philippe Viard, « Le soin-communicant », Communication et organisation [En ligne], 51 | 2017, mis en

ligne le 01 juin 2020, consulté le 02 janvier 2021. URL : http://journals.openedition.org/

communicationorganisation/5578; DOI : https://doi.org/10.4000/communicationorganisation.5578

(C) Presses universitaires de Bordeaux 


\section{Le soin-communicant \\ Un espace de médiation en vue de la construction \\ d'une professionnalité soignante}

\section{Philippe Viard'}

\section{Le monde de la santé confronté à de nouveaux enjeux}

La santé n'est pas un secteur d'activité qui échappe aux problématiques contemporaines de l'économie, des finances et, plus largement, du changement structurel de la société dans laquelle l'individu occupe une place nouvelle. Gilles Lipovetsky note un paradoxe dans cette " civilisation du désir » (Lipovetsky 2006 : 21) née d'une nouvelle modernité : "d'un côté plus que jamais, les individus prennent soin de leur corps, sont obsédés d'hygiène et de santé, obéissent aux prescriptions médicales et sanitaires. D'un autre côté prolifèrent les pathologies individuelles, les consommations anomiques, l'anarchie des comportements » (Lipovetsky $2004: 76$ ).

Le soin est dès lors, directement affecté par ce contexte sociétal qui induit des décisions particulières au sein des politiques publiques en matière de santé. Celles-ci sorganisent en prenant en compte le vieillissement de la population française et le contexte lié à l'offre et à la demande de soins en santé. De surcroît, les problèmes économiques impactent durablement le système de santé qui reste une préoccupation importante en France. Dans l'analyse qu'en fait Joseph E. Stiglitz, prix Nobel d'économie, le Produit Intérieur Brut (PIB) mesure l'état de notre système de santé. Il fait le constat que « si le PIB par habitant des États-Unis paraît plus élevé que celui de la France et du Royaume-Uni, c'est en partie parce que notre système de santé est moins efficace. Nos dépenses de santé sont très supérieures et nos résultats très inférieurs » (Stiglitz 450).

Au regard de cette approche multidisciplinaire de la santé, quelle légitimité $\mathrm{y}$ a-t-il à choisir le soin, et particulièrement la relation de soin, comme objet de recherche en Sciences de l'Information et de la Communication (SIC) ? L'hypothèse générale de notre travail de recherche est que la communication

1 Docteur en Sciences de l'Information et de la Communication, cadre de santé, formateur à l'IFSI du CHU de Dijon, laboratoire CIMEOS (EA 4177), Université de Bourgogne ; viardphil@gmail.com 
constitue un élément essentiel du soin. Si elle est enseignée dans les organismes de formation infirmière, c'est plus particulièrement comme un outil au service de la pratique et comme un besoin de la personne soignée à prendre en compte. La présence du concept de communication dans l'univers des significations du monde du soin ne suffit pas à justifier l'appartenance du soin au champ épistémologique des SIC. Le soin répond toutefois aux quatre principes retenus par Jean Davallon, qui permettent de construire un objet de recherche et d'en aborder la construction d'un point de vue infocommunicationnel (Davallon 30-37) :

- Le premier principe est la prise en compte du «lestage technosémiotique ». Sur ce point, la relation de soin est conforme à ce premier critère car elle est, en tant que telle, un élément technique au sens d'un objet pouvant être objectivé et observé. Elle n'est pas une abstraction ou une simple représentation, mais bien un "composite » complexe de faits de communication, d'échanges et d'interactions.

- Le deuxième principe prend appui sur la nécessité de réaliser un recueil de données en fonction du principe de "réflexivité ", c'està-dire d'adaptation des techniques à l'objet recherché. C'est le cas de notre corpus de recherche, construit progressivement pour permettre de comprendre la relation de soin et la manière dont elle s'inscrit dans une dimension corporelle.

- "L'échelle d'observation " est le troisième principe. Pour notre recherche, cette échelle d'observation est celle des acteurs du soin dans la mesure où ce sont eux qui rapportent des situations vécues et analysables.

- Ces dernières nous servent de support pour atteindre un " degré d'abstraction » qui, selon le dernier principe de Jean Davallon, est relatif à la conceptualisation et à la modélisation d'un travail de recherche.

Ces trois éléments, ainsi décrits, nous permettent d'adopter une posture "d'interdisciplinarité focalisée » (Charaudeau 222). En effet, si l'éclairage de la psychologie et plus particulièrement de la psychologie sociale nous aide à décomposer et à comprendre les situations de soins, il nen demeure pas moins que c'est au bénéfice de l'analyse des formes communicationnelles dans le soin, des effets de sens des pratiques et des logiques de communication des soignants. Nous nous inscrivons dans la perspective d'une lecture communicationnelle des représentations, au service du décryptage des phénomènes sociaux au sens décrit par Yves Jeanneret d'êtres culturels « complexes qui associent des objets matériels, des textes, des représentations et qui aboutissent à l'élaboration et au partage d'idées, d'informations, de savoirs, de jugements. » (Jeanneret 16)

\section{Les quatre piliers du soin}

Problématiser un sujet comme le soin et plus précisément sa dimension communicationnelle, nécessite de réaliser une contextualisation solide 
afin de donner une assise suffisamment stable et valide à l'analyse et à la compréhension des logiques des acteurs concernés. À ce sujet, quatre éléments sont structurants :

- le territoire du soin, dans sa dimension sensible ${ }^{2}$ et symbolique, en particulier en ce qu'il constitue un espace entre une relation ordinaire au corps et une organisation construite de philosophies et théories $d u$ soin. À partir de cette double perspective, nous pensons qu'il existe une réelle culture du soin au sein du groupe social des soignants. Il convient donc, comme nous le propose Anne-Marie Laulan, d'aborder ce territoire du soin en prenant en compte sa contextualité et sa complexité car " toute notion de territoire renvoie à celle du pouvoir et donc à celle de l'exclusion" (Laulan).

- la pratique du soin, inscrite dans un premier temps dans une histoire fortement influencée par le monde spirituel et religieux, puis dans un second temps, rendue dépendante des changements opérés par les découvertes scientifiques ;

- l'offre de soin, définie à la fois par des réformes successives et des attentes des usagers, en particulier du fait d'un vieillissement de la population modifiant les demandes et les attentes en santé ;

- la formation au soin qui accompagne les changements sociétaux et organisationnels en recherche d'adéquation avec les attentes des futurs professionnels.

\section{Le soin: un processus communicationnel complexe}

L'une des premières questions qui se pose lorsque l'on évoque le soin est la place des processus communicationnels dans un espace de médiation de la relation de soin ou par la relation de soin. Actuellement, le soin est pensé comme la réponse à un besoin, une attente, une nécessité et plus simplement, à une prescription. Est-il possible d'imaginer le soin comme un processus de médiation, un dispositif communicationnel où interagissent les acteurs du soin, c'est-à-dire le soigné, le soignant, la famille, les bénévoles, ... ?

Plus précisément, ce processus de médiation aurait-il une forme qui, en fonction des logiques qui l'organisent, ferait émerger et mettre en jeu des valeurs, des représentations, des productions identitaires propres au groupe des soignants et, en tant que telles, des problématiques liées à des échanges de significations quant aux objectifs et à la pratique du soin ? Ce questionnement s'inscrit dans la complexité des nouveaux modèles organisationnels de la société. L'analyse que nous faisons des logiques d'acteurs, et en particulier

2 Nous retiendrons comme définition du sensible la faculté d'appréhension du monde par le corps, caractéristique du rapport au «monde de la vie » (Schütz, 1932 ; Perreau, 2010). Si toutefois, "l'expérience du terrain est une expérience de partage du sensible » (Laplantine, 2005), le sensible, en lui-même, est cette capacité à percevoir les "phénomènes subjectifs corporels» (Bois, 2011), les formes naturelles de l'expérience communicationnelle, les espaces de relation inter-individus. 
de leurs représentations liées à l'action, nous interroge sur cette progressive évolution d'une pensée fondée sur l'acte de soin vers un modèle construit à partir de processus communicationnels complexes. Il est dès lors possible d'imaginer le soin comme un modèle d'une interrelation dynamique, basée sur une co-construction du sens par les acteurs en situation de soin. Rosette et Jacques Bonnet suggèrent que « la découverte de la lente émergence de l'idée de co-construction du sens, ainsi que du concept d'interaction et de ses effets [...] invite la mise en dialogue de l'évolution des modèles organisationnels du travail et des modèles d'analyse de la communication humaine.» (Bonnet 30). La relation de soin fait partie de cette lente mutation organisationnelle « du simple au complexe ».

Par ailleurs, notre réflexion ne peut ignorer l'un des éléments centraux du soin : le corps. Les travaux sur le concept de « corps communicant» (Melenec, Martin-Juchat) ont été à l'origine de notre questionnement et, en particulier, lorsque leurs auteurs invitent la communauté scientifique à " construire un champ de recherches sur les pratiques du corps » (Melenec, Martin-Juchat 13). Le soin est l'une de ces pratiques et nous sommes amenés à constater que, dans la pratique soignante, il est souvent plus simple de traiter le corps seul que le soigné dans sa globalité. Nous souhaitons considérer le corps hors du dualisme corps-physique/corps-psychique et, à l'instar d'Emmanuel Mounier, considérer que l'Homme vit dans une « ambiance corporelle " (Mounier 117).

La place du corps dans le soin constitue donc l'une des parties essentielles de notre recherche. Il ne s'agit pas, de ce point de vue, d'évaluer la réalité du corps malade ou d'appréhender le corps comme support du soin. Nous interrogeons le concept de corps en tant que réalité vivante, perçue à la fois chez le soigné et le soignant. En ce sens, nous nous intéressons plus particulièrement aux articulations des différents espaces du corps et des corps et, à travers elles, aux rencontres et aux distances représentationnelles chez les acteurs concernés.

\section{Un terrain pluriel pour une analyse compréhensive}

Le terrain que nous avons construit pour cette recherche nous a permis l'appréhension de la réalité soignante plurielle et multiforme. Ce terrain existet-il en tant que corpus saisissable ? Selon nous c'est le cas, dans la mesure où il est le produit formalisé d'un reconstruit intellectuel, aux limites définies par notre problématique et nos hypothèses. Comme le dit Yves Winkin à propos de l'anthropologie de la communication, elle « ne se définit plus par ses objets, mais par le regard qu'elle porte sur les objets qu'elle décide d'investir, par les rapports qu'elle entretient avec les acteurs sociaux, par l'écriture qu'elle utilise pour rendre l'expérience vécue »(Winkin 114).

Dans le cadre de la création du terrain propre à la recherche, il est nécessaire de préciser que si le monde de la santé fait partie de notre quotidien, nous avons veillé à réaliser cet effort de distanciation qui prévaut à tout travail de recherche. Ce positionnement face au terrain est une règle que nous nous 
sommes fixée, tant il est important pour le recueil des données du corpus, d'adopter un positionnement non apriorique. Philippe Amiel le définit comme une posture d'indifférence ethnométhodologique, « analogue à ce que Weber appelle "neutralité axiologique", et que Schütz désigne par "attitude de l'observateur désintéressé". Elle traduit une règle méthodologique de la phénoménologie husserlienne : la suspension du jugement "mondain", la "mise entre parenthèses", l'épochè - voie royale pour l'accès "aux choses-mêmes" » (Amiel 72).

Cependant, nous avons conscience que nos propres représentations sont agissantes sur notre relation au corpus et à l'analyse : " Il serait naïf de penser qu'il est possible d'extraire la démarche scientifique du social (Khun, 1962). Encore davantage, quand la recherche porte sur l'humain, quand il s'agit de domaine où l'intime et l'affect jouent (la sexualité, l'homosexualité, le genre, l'euthanasie, les croyances religieuses, etc). Comment imaginer que le chercheur qui travaille ces domaines ne soit confronté à son propre vécu ? " (Galinon-Melenec)

Cette posture téléologique nous a obligés à utiliser des informateurs, lorsqu'il était nécessaire d'appréhender le réel du soin en actes. Nous y avons relevé les indices de la communication en situation par ceux qui réalisent le soin. Comme nous le propose Daniel Bougnoux : « opérateur de continuité, d'attachement et de contact, l'indice est le lest irremplaçable de tous les cours ; de même, les soins du corps sont au cœur du social contemporain. L'arc-enciel des communications indicielles est le vecteur du sens, s'il est vrai que ne "fait sens" pour chacun que cette information qu'il peut relier à son corps, ou à l'organisation vivante en général. » (Bougnoux 1998 : 70)

Notre choix méthodologique a été celui de réaliser cinq analyses différentes. Chacune d'entre elles est construite à partir du principe selon lequel « l'action communicationnelle ne met pas en relation le sujet et l'objet (couple technique), mais le sujet avec le sujet (couple pragmatique). C'est l'homme agissant sur (les représentations de) l'homme par le détour des signes » (Bougnoux 1996 : 20).

Nous avons adopté une approche compréhensive des logiques mises en place par les acteurs à partir d'une analyse « de la clinique de l'activité [(Clot, 1999)] ou de l'événement communicationnel » (Brassac, Fixmer 90). Nous avons abordé la notion de logique sous l'angle sociocognitif, comme étant celle qui « synthétise une manière de se situer, de lire, d'interpréter, de réagir et d'intervenir, qui est certes plus ou moins homogène, stable et durable, mais qui témoigne du rapport Homme/travail/organisation, rapport en tension et en mouvance permanente» (Bonnet 2003 : 151).

Le terrain que nous avons construit s'organise à partir de cinq lieux différents :

- Le premier est celui des médias publics et, en particulier,celui de l'affichage médiatique de femmes qui représentent symboliquement le soin. Le 
choix de ces personnages exemplaires, dans ce qu'ils communiquent d'images symboliques, répond au besoin de connaître l'environnement du soin et la représentation publique du soignant. Nous avons réalisé une lecture macro-communicationnelle de ces images médiatiques afin d'en déduire la représentation de sens commun du soin et de ce que ces femmes véhiculent comme prescription au modèle soignant.

- Le deuxième lieu est celui du soin et en particulier dans le domaine $d u$ confort et du bien-être généralement appelé « toilette ». Une analyse de cet acte nous permet de figurer le sens de ce soin, acte emblématique de l'activité soignante. Bien au-delà d'un simple soin technique, la toilette permet d'appréhender la dimension meso-communicationnelle de l'activité soignante par ses implications sensorielles, sensibles et symboliques. Au-delà de toute tentative de définition, la toilette est sans doute devenue une catachrèse du soin.

- Le troisième lieu est celui de situations de soins rencontrées par des soignants et mises en récit. Toutefois, si ces situations ont été réellement vécues, elles demeurent le produit d'un discours a posteriori, révélateur des représentations des acteurs. Cependant, comme le décrit Lise Renaud, «si les représentations sont souvent des productions discursives, elles peuvent aussi ne pas être conscientes ou exprimées (notamment lorsque l'objet de la représentation est le corps ou la santé), et c'est alors au travers des pratiques qu'elles peuvent être appréhendées » (Renaud, Thoer 351). C'est pourquoi, en l'accompagnant dans son récit par un entretien, chaque soignant a pu décrire la situation qu'il a vécue : " pour le connaître [le vécu de l'acteur] afin de le modéliser et de le perfectionner, il faut que celui qui l'a vécu, comme celui qui cherche à s'en informer le reconnaisse »(Vermersch 30). Même si pour le soignant son activité peut « aller de soi » en tant qu'acteur social, il " peut et il est le seul à pouvoir le faire aussi parfaitement, décrire, commenter ses actions, leur pourquoi, leur comment, et jeter sur celles des autres membres un éclairage significatif. »(De Luze 23). À partir de ce recueil de situations, nous avons réalisé une analyse micro-communicationnelle d'activités professionnelles types et dans le cadre d'une étude des représentations.

- Le quatrième lieu est le récit de vie d'une personne suisse, qui a décidé d'accueillir à son domicile une personne âgée dépendante non-membre de sa famille. Cette démarche nous a interrogés sur le sens que pouvait révéler cet engagement de prise en charge d'une personne âgée hors d'un cadre professionnel. Ce récit de vie a fait l'objet d'une analyse diachronique et compréhensive afin de dégager les caractéristiques d'un engagement soignant par une personne "non-soignante ${ }^{3}$. Le réel mit en récit par l'auteur de cet accueil, permet de faire émerger une réalité vécue et

3 Nous utiliserons l'expression "non-soignant » pour signifier le caractère non-professionnel de l'activité de soin. 
perçue où peuvent apparaître de nombreuses significations : «le réel est ce qui est au-delà des mots, au-delà des signes : au-delà en définitive de la limite qui fonde l'espace symbolique de la communication. » (Lamizet 74).

- Le cinquième lieu est celui de l'espace monographique que représente le programme de formation infirmière. Cet enseignement est proposé par les Instituts de Formation en Soins Infirmiers (IFSI) qui s'appuient sur trois référentiels nationaux ${ }^{4}$. Ils nous ont permis d'aborder la question suivante : en quoi la personne âgée est-elle prise en compte, au sens de " en quoi lui attribue-t-on de la valeur ", à la fois au sein d'unités dénommées " sciences humaines ", "biologiques et médicales » et " sciences infirmières et d'intégration des savoirs "? Cette dernière analyse nous a permis de construire un socle de préconisations de nature stratégique, dans le contexte d'harmonisation des formations de santé en vue d'une collaboration interprofessionnelle plus efficiente au service du patient.

\section{Le soin-communicant: vers un concept intégrateur}

L'analyse de notre corpus nous amène donc à concevoir le concept de soin-communicant, un modèle à part entière, permettant en lui-même une compréhension différente du soin, de sa pratique et des logiques portées par ses acteurs. Cette modélisation est rendue possible dans la mesure où penser le soin en termes d'espace, d'interface et de médiation ne vient pas contredire les modèles précédents, mais plutôt ouvrir un espace de compréhension différent. La dimension communicationnelle du soin permet de le concevoir non pas comme une structure rigide, mais comme un processus, c'est-à-dire qu'il est à la fois structuré dès sa conception et en même temps modelé par l'activité elle-même. L'espace qu'il occupe vient alors en enchevêtrement des espaces de signification du soignant et du soigné. Chaque intervenant dans cette relation bénéficie d'une médiation, notamment celle du corps, dont la définition que nous retiendrons sera qu'elle est « un moyen pour l'individu de prendre conscience de ses opinions, de ses représentations, de ses croyances » (Martin-Juchat 2006 : 94).

Nous utilisons le terme médiation au sens où il permet effectivement de comprendre ce qui est en jeu dans le soin et particulièrement les dimensions rationnelles et sensibles en rapport avec la représentation du réel pour l'acteur (Bonnet 2003 : 220). Dans ce cadre, le concept de soin-communicant permet effectivement de passer d'une logique de soin unique à celle d'un soin élaboré comme un processus de médiation dans lequel sont impliqués soigné et soignant. Se situer dans la relation de soin et identifier l'espace du soincommunicant peut permettre au soignant d'entreprendre une activité réflexive

4 La réforme de la formation infirmière en 2009 organise les enseignements à partir de trois référentiels : un référentiel d'activité, un référentiel de compétences et un référentiel de formation. 
et de développer une professionnalité au regard de sa réalité professionnelle et de la construction de son identité personnelle (Dubar 1992, 2010). Il pourra expérimenter le sens du complexe (Bonnet 2003), dans ce que le soin implique d'éléments relationnels, identitaires, organisationnels, technologiques et communicationnels.

Si le soin est vu comme un processus d'une médiation communicationnelle, il interroge la temporalité du soin. En effet, il ne s'agit pas de le penser comme une succession d'actes uniques, mais de l'inscrire dans une perspective d'attention singulière au soigné, dans une dimension communicationnelle forte. Notre analyse a montré que cette dimension est revendiquée par les soignants eux-mêmes. Leur demande ne consiste pas à vouloir mobiliser plus de temps pour le soigné mais à être plus attentif à ce qu'il est, ce qu'il représente.

\section{Le corps-monde, espace d'une géographie relationnelle}

Pour appréhender plus précisément cet espace du soin-communicant, nous proposons de penser le corps dans une dimension élargie où le corps est à la fois source, lieu et objet de la communication. À l'instar de ces villes-monde (Baudrel 1979) dont on parle désormais, nous proposons une analogie en définissant un corps-monde, organisé par une morphologie sociale et situé dans sa propre géographie relationnelle. Le corps-monde doit s'organiser autour de ses propres enjeux : un multiculturalisme, une prise en compte des implications identitaires variées, une mise aux normes sociales, une sécurité interne. Chaque individu a ses propres bidonvilles, relégués à la périphérie de son corps, ses propres lieux communautaires, ses ressources et ses nourritures.

- Le corps-monde est source de la communication. La présence du corps, réelle ou symbolique, lui donne un statut de source de la communication en tant qu'élément à l'initiative du mode de la médiation. En absence de corps, il ne peut y avoir de soin ; sans corps, il ne peut y avoir de vie. Cette affirmation semble une évidence, il s'agit pourtant d'un point fondamental de notre travail de recherche. L'analyse montre que, quel que soit le référentiel adopté par le soignant, quel que soit le type d'intervention ou la logique qui le préside, il n'y a pas de soin sans corpsmonde. Qu'il s'agisse de soin physique ou psychique, de soin relationnel ou technique, de soin attaché à un organe ou à un processus, de soin biologique ou psychiatrique, ou de tout autre champ référentiel, le corpsmonde est toujours présent.

- Le corps-monde est lieu de la communication, car il procède de la même intention que le contexte dans lequel il existe. Notre analyse fait apparaître ce lien très étroit entre les valeurs de l'individu et sa capacité à être en communication; dans le cadre de la référence au corps-monde, nous pourrions aller jusqu'à dire à être communication dans une vision d'une impossibilité à ne pas communiquer (Watzlawick 1967). 
- Le corps-monde est objet de la communication car il est, entre autres, support des modes opératoires des acteurs, des évocations qu'il induit et de l'éclairage des notions et des concepts qu'il suscite. L'analyse permet de démontrer que le corps-monde est à la fois représenté et représentation et qu'il donne aux acteurs une justification de la nécessité d'une communication. Par nature, le corps-monde est lobjectif du soignant par une participation effective et réelle au soin. En tant qu'objet de communication, le corps-monde est terreau de la médiation, il ouvre des espaces de sens pour la compréhension du monde qu'il crée et qui le crée.

\section{Corps-monde et soin : une transaction du sens}

Qu'en est-il alors du lien entre cette notion de corps-monde et du soin ? L'ensemble de nos analyses permet de confirmer l'hypothèse selon laquelle il existe dans le soin, un lieu-corps au sein duquel le sujet interagit avec son contexte. De ce point de vue, le corps est un objet du rapport social de l'Homme à son environnement. Dans le même temps, il est le lieu du sens que Daniel Bougnoux qualifie de complexe : « il est clair que le sens commence avec la sensation, soit au niveau de ce qui touche indiciellement le corps. Avant de relever du jugement de la re-présentation, le sens est d'abord sensible, organique, il s'enracine dans le pacte inextricable du moi et du monde, du dedans et du dehors, de l'observateur et de l'observé » (Bougnoux 1998 : 238). Pour le soin, ce lien entre observateur et observé peut sembler une évidence lorsque le soignant observe le corps du soigné et l'ausculte à la recherche d'indices cliniques utiles à son activité. Mais l'observateur est aussi le malade, qui voit le corps du soignant et qui cherche le sens de ce que le professionnel est et fait.

Le soignant et le soigné possèdent donc chacun leur propre corps-monde. Le corps-monde est ainsi un espace corporéisé dans les limites d'un corps physique (un corps-chair) et en même temps, corps contenu dans un espace culturel et social (un corps-sensible). Le corps-chair ne peut être nié car il procède d'une réalité non symbolique. Le corps-sensible, lui, est inscrit dans les perspectives culturelles du sujet appartenant à un groupe social qui l'accueille et l'organise.

Le soin est donc un ensemble de gestes, de mouvements, de contacts qui sordonnent en impliquant le corps du soignant et celui du soigné. Pour Christophe Genin, le corps-monde serait l'approche singulière des ressentis sensoriels du corps « au contact du monde. [...] le corps-monde n'est ni que corps, ni que monde »(Genin, Leroux, Lontrade 106), un corps de chair interfacé avec son environnement par le corps. Cette vision chorégraphique du rapport du corps au monde corrobore l'idée, proposée par Fabienne Martin-Juchat, que "l'émotion est une mise en mouvement de la chair, la parole est une expression corporelle » (Martin-Juchat 2010). 
Ce corps-monde ainsi défini est une notion qui permet de clôturer le champ de ce qui appartient au sujet en tant qu'individu et de ce qui relève du contexte en tant que substrat social et identitaire. À ce titre, si la relation de soin n'existe qu'au travers de la présence d'un corps réel, son statut est également celui d'un corps média comme le propose Fabienne Martin-Juchat : " Définir le corps/média comme un lieu d'interactions, de rencontres entre différents systèmes, implique aussi l'idée que l'interaction interpersonnelle est toujours médiatisée par des corps qui sont porteurs de discours collectifs, véritables intermédiaires, artefacts semi-technologiques entre individus, comme entre individus et collectifs.»(Martin-Juchat 2006: 149). Le corps-monde est ainsi, notamment dans le soin, un espace médiatisé, un dispositif propre à la rencontre des corps-chair et des corps-sensibles, rencontre propre à la relation interpersonnelle.

La problématique de la relation de soin n'est donc pas celle d'une distance sensorielle, mais celle d'une limite réelle et symbolique qui oblige le soignant à la réflexion et à l'analyse de sa pratique. Il n'y a pas de proxémie une et universelle, mais une prise en compte d'un espace que peut occuper la maladie. C'est toute la difficulté de la rencontre avec l'altérité et de l'évaluation de ce que la maladie impacte pour le malade. La limite du corps-monde n'est pas de l'ordre d'une rupture, mais plutôt un espace de transition voire de transaction du sens.

\section{Quel mode d'intervention pour le soignant?}

L'analyse de notre corpus nous révèle que le soignant agit dans la relation avec son corps-monde. On peut en effet considérer que la dimension soignante de l'acteur appartient à ce qui le constitue, en particulier dans sa dimension de socialité et d'intériorité, de culture et d'identité. Être soignant ne participe pas de la même logique qu'avoir une activité soignante. Être soignant est ancré durablement dans une identité personnelle, construite par ce qu'est l'acteur, en tant qu'individu, fruit d'un parcours et d'une histoire de vie qu'il met luimême en récit. L'acteur devient soignant parce qu'il se reconnaît lui-même soignant. Mais il l'est également parce que le soigné, lui aussi, le reconnaît soignant. Les normes et injonctions sociales participent à la création de l'identité soignante. Mais encore faut-il l'accepter et comme le dit Emmanuel Levinas : " chaque individu est virtuellement un élu, appelé à sortir, à son tour - ou sans attendre son tour - du concept du Moi, de son extension dans le peuple, à répondre de responsabilité : moi, c'est-à-dire, me voici pour les autres, à perdre radicalement sa place - ou son abri dans lêtre » (Levinas 233).

Notre analyse montre à quel point les situations de soins entraînent un investissement important des soignants, tant sur le plan d'un engagement professionnel que sur un plan plus personnel. Si l'on considère que ces deux éléments font partie intégrante du corps-monde, l'implication du soignant dans une situation ne relève pas d'une attitude à adopter vis-à-vis de telle 
ou telle situation de soin, mais d'un engagement construit à partir de valeurs soignantes.

Si corporéité et axiologie construisent une dimension du soin, elles lui donnent sens dans la dimension de la relation, qui est présente dans tout acte de soin en partant d'un postulat que ce dernier n'est possible que s'il y a relation entre un corps physique et un corps social. Cet espace relationnel créé impacte les logiques soignantes, mais également la perception des personnes soignées. La mesure de l'implication relationnelle est souvent découverte au sein même de l'activité et de la médiation au soin. Les acteurs développent des habiletés qui, au sein de l'organisation soignante, permettent d'accroitre les savoirs collectifs partagés. Il s'agit d'en connaître l'origine, déduite des théories et concepts ou induite par l'activité de terrain. La relation de soin est appréhendée par le biais de l'acte de soin, dans une perspective fonctionnaliste, qui met en avant efficacité et protection. Le soin est construit autour de modèles répondant d'abord aux exigences médicales dont la dimension communicationnelle est celle de moyens permettant l'atteinte des objectifs. Une approche communicationnelle aura pour visée d'appréhender le soin à travers la posture soignante, mais également d'un point de vue plus général, au regard de l'ensemble des acteurs de la relation, acteurs engagés dans un système de significations où, pour reprendre la célèbre métaphore de Goffman, engagés dans l'orchestre de la pratique soignante.

\section{Médiation de soin et professionnalité}

L'une des perspectives notre travail et de permettre, à partir du concept de soin-communicant, de rendre visible des problématiques qu'un soignant (ou étudiant en soin) aura à analyser et à s'approprier. Cette réflexivité est nécessaire pour la construction de son identité professionnelle. Cette élaboration à partir de ses pratiques de soins est possible si le soignant a délimité l'espace du concept de soin-communicant. En effet, il s'agit pour lui de différencier une analyse de la pratique en tant que telle et une supervision qui relève d'un autre type d'investigation, plus personnelle et plus intime.

L'analyse d'une médiation de soin vise donc à favoriser la création d'une identité professionnelle par un processus de construction du sens sur une praxis qui met en jeu le corps-monde du soignant. Comme le signale Thierry Blin dans une description de la thèse schülzéenne des "perspectives réciproques ", «dans la relation de face-à-face, jéprouve directement, immédiatement, l'autre dans sa corporéité vivante »(Blin 21). L'analyse d'une médiation de soin ne se conçoit pas comme un simple exercice intellectuel et linguistique, il convient de comprendre, comme le dit Bourdieu, « ce que parler veut dire »(Olivesi 65) tout en prenant en compte l'activité réelle de soin dans une contextualisation sociale. 
En effet, le monde s'organise autour de l'acteur et l'acteur lui-même en a une perception par le biais de son corps-monde. C'est cette spatialité qui permet d'entrevoir qu'il saisit son positionnement, non pas par imposition d'une signification d'origine sociale, mais par une posture individuelle de compréhension et de transformation de son propre corps-monde, à la fois monde social individualisé et corps-chair, réalité première. Le corps-chair est là aussi présent comme espace du sens, un « un corps-objet [...] pensé comme corps tout court, comme espace d'abord et uniquement " (Ricœur 205). Le corps-monde contient le langage et l'énonciation de la pratique, il peut permettre de faire une analogie entre analyse d'une médiation de soin et d'une modalisation au sens goffmanien d'une " transformation qui ne se cache pas»(Goffman 290).

\section{La perspective d'un soin engageant}

La conclusion de notre recherche permet de confirmer que le corps, comme nous le propose Florence Vinit, est « au cœur du soin »(Vinit 2007). À partir de l'angle de perception des soignants, le concept de corps peut ainsi être élargi à une notion plus large et plus globalisante, le concept de corps-monde, tel qu'il permet d'intégrer le soin dans une dimension communicationnelle forte.

C'est ainsi que la dialogique soignant/soigné organise et autorise une médiation relationnelle qui en elle-même peut être au bénéfice de la thérapeutique. Il existe entre les deux actants du soin un lien dont la communication est le vecteur et le levier.

Mais il est essentiel de prendre en compte que : «le toucher nécessite l'intervalle d'un entre-deux. C'est le fait de ma corporéité qui me permet de toucher le monde, de me toucher comme être de corps " (Vinit 157). Notre recherche confirme que le soigné et le soignant sont membres de cette relation, à part égale, chacun étant à la fois touché et touchant, au cœur d'un système d'échanges signifiants dans un ensemble que nous avons nommé le soin-communicant.

Le soin est un processus évolutif et complexe, ce qui fait toute la difficulté d'adapter les méthodes d'enseignement en institut auprès d'étudiants qui recherchent des réponses factuelles, des modes opératoires généraux, des modèles de pratiques protocolisés et immédiatement applicables.

Le modèle que nous proposons et de nature à permettre à l'acteur d'investir la dimension de sa professionnalité, tout à la fois individu soignant, impliqué comme acteur du soin et acteur social, représentant et créateur d'actes socialement identifiés et engagés. Le soin engage celui qui le fait, celui qui le reçoit, et reste un enjeu majeur de notre société. «Vivre est un art » (Vasseur 2014) nous propose Alain Vasseur. Parce que vivre participe d'un apprentissage, 
d'un métier, d'une ouverture au monde... Et, comme le souligne cet auteur, la mise en « œuvre » de sa propre vie s'alterne entre intériorité et partage. C'est sans doute l'un des objets du soin, de l'engagement soignant...

\section{BIBLIOGRAPHIE}

AMIEL Philippe, 2010, Ethnométhodologie appliquée. Eléments de sociologie praxéologique, Paris, Presses du Lema, 207p.

BLIN Thierry, Alfred SCHÜTZ, 1998, Éléments de sociologie Phénoménologique, Paris, L'Harmattan, 158 p.

BONNET Rosette, BONNET Jacques, 2003, Nouvelles logiques, nouvelles compétences des cadres et des dirigeants. Entre relationnel et sensible, Paris, L'Harmattan, 253 p.

BOUGNOUX Daniel, 1998, La communication par la bande : Introduction aux sciences de l'information et de la communication, Paris, La Découverte, $276 \mathrm{p}$.

BOUGNOUX Daniel, 1996, «Qu'est-ce que la communication ?» $M E I, \mathrm{n}^{\circ} 4$, p. 17 22.

BRASSAC Christian, FIXMER Pierre, 2007, «La production du sens en organisation : un processus cognitif situé et distribué. » dans L. BONNEVILLE et S. GROSJEAN, Repenser la communication dans les organisations, Paris, L'Harmattan, $300 \mathrm{p}$.

CHARAUDEAU Patrick, 2010, « Pour une interdisciplinarité « focalisée » dans les sciences humaines et sociales ", Questions de communication, n ${ }^{\circ} 17$, p. 195-222.

DAVALLON Jean, 2004, "Objet concret, objet scientifique, objet de recherche ", Hermès, n 38 , p. 30-37.

DE LUZE Hubert, 1997, L'ethnométhodologie, Paris, Éditions Economica, 111 p.

GALINON-MÉLÉNEC Béatrice, MARTIN-JUCHAT Fabienne, 2014, Du « genre » social au « genre » incorporé : Le « corps genré » des SIC. In Revue française des sciences de l'information et de la communication [En ligne]. n 4, [consulté le 23 décembre 2016]. Disponible sur : http://rfsic.revues.org/857

GENIN Christophe, LEROUX Claire, LONTRADE Agnès, Juger l'art? Paris, Publication de la Sorbonne, 2009, $196 \mathrm{p}$.

GOFFMAN Erving, Frame Analysis: An Essay on the Organization of Experience, New York, Harper an Row, 1974, 600 p.

JEANNERET Yves, Penser la trivialité. Volume I: la vie triviale des êtres culturels, Paris, Hermes Science Publications, 2008, 266 p.

LAMIZET Bernard, Les lieux de communication, Wavre, Pierre Mardaga Éditeur, 1995, $343 \mathrm{p}$.

LAULAN Anne-Marie, Liens et lieux. Séminaire du laboratoire CIMEOS, "L'intelligence territoriale et les lieux - Quel(s) rapport(s) avec les territoires ? ", Dijon, le 25 juin 2014.

LEVINAS Emmanuel, Autrement quêtre ou au-delà de l'essence, Londres, Kluwer Academic Publishers, 1991, 243 p.

LIPOVETSKY Gilles, Le bonheur paradoxal, Paris, Gallimard, 2006, 377 p.

LIPOVETSKY Gilles, Les temps hypermodernes, Paris, Grasset, 2004, 112 p. 
MARTIN-JUCHAT Fabienne, Penser le corps affectif comme média, mémoire d'Habilitation à Diriger des Recherches, Université de Bourgogne, 2006, Tome 1, 240 p.

MARTIN-JUCHAT Fabienne. Quelle place aux émotions et aux mouvements dans la transmission du savoir? Conférence-performance en collaboration avec Etienne QUINTENS (danseur). Lyon : Colloque Marcel Jousse [En ligne]. 2010, [consulté le 15 janvier 2017]. Disponible sur : http://www.academia.edu/3535817/Quelle_place_ aux_emotions_et_aux_mouvements_dans_la_transmission_du_savoir_Texte_sur_la_ performance_lors_du_colloque_Marcel_Jousse

MELENEC Béatrice, MARTIN-JUCHAT Fabienne, Le corps communicant - XXIe siècle, civilisation du corps? Paris, L'Harmattan, 2007, $242 \mathrm{p}$.

MOUNIER Emmanuel, Traité du caractère, Editions du Seuil, 1946, 796 p.

OLIVESI Stéphane, La communication selon Bourdieu, Paris, L'Harmattan, 2005, 92 p.

RENAUD Lise, THOER Christine, mai 2007, «Éditorial », Santé Publique, vol. 19, p. 351-352.

RICEUR Paul, Philosophie de la volonté, tome 1. Le Volontaire et l'Involontaire, Paris, Seuil, 2009, $624 \mathrm{p}$.

STIGLITZ Joseph E., Le triomphe de la cupidité, Paris, LLL, 2010, 474 p.

VASSEUR Alain, Introduction, Colloque "L'habitat fragile, l'espace intime, l'espace refuge... Abri réel et symbolique, petit radeau de vie dans le grand mouvement du monde ", Itinéraires Singuliers, Dijon, 13 mars 2014.

VERMERSCH Pierre, mars 2005, Aide à l'explicitation et retour réflexif, Journal de l'association GREX, n' 59, p. 26-31.

VINIT Florence, Le toucher qui guérit. Du soin à la communication, Paris, Belin, 2007, $203 \mathrm{p}$.

WINKIN Yves, «Vers une anthropologie de la communication ? » dans Ph. CABIN et J.-F. DORTIER, La communication : état des savoirs, Paris, Sciences Humaines, 2008, $400 \mathrm{p}$.

Résumé : Dans le contexte français des formations en soins infirmiers, cette thèse propose de concevoir le soin comme un processus de médiation et déchange de significations entre les acteurs concernés, notamment soignés et soignants. Les processus communicationnels identifiés ici mettent en jeu des valeurs, des représentations et des positionnements identitaires qui concourent à l'élaboration et à l'expression d'une professionnalité soignante. Le corpus de cette recherche est organisé à partir d'images médiatiques, d'activités de soin, de récits relatifs à des situations professionnelles, d'un récit de vie et d'une monographie. Ces cinq sources de données permettent une approche compréhensive des formes communicationnelles mobilisées dans le contexte du soin, des effets de sens relatifs aux pratiques observées ainsi que des logiques d'intervention et de relation des personnels soignants. Le concept de corps-monde est proposé comme modèle intégrateur des rencontres et des distances relationnelles co-construites par les acteurs du soin. Enfin, pour répondre aux questionnements d'ordre stratégique auxquels se trouvent confrontées les organisations de santé dans le cadre des nouvelles pratiques de 
soin et des évolutions de la formation infirmière, la notion centrale de soin-communicant est installée afin de définir le soin comme un système complexe d'échanges signifiants. Ce concept tente de formaliser les espaces de médiation propres à la construction d'une professionnalité soignante pertinente et cohérente au regard des nouveaux enjeux de la formation en soins infirmiers.

Mots-clés : soin, formation, corps, médiation, valeurs, identité, activité.

\begin{abstract}
In the french context of nursing care teaching, this thesis aims at conceiving nursing as a process of mediation and communication notably between especially patients and care givers. The identified communication process involving values, self-identification and representation help to build up and express the caring professionalism. The background documents are organized from 5 types of data which are media focuses, caring acts, professional situation reports, life stories and monographs. These data allow the comprehension of the communication items which are used in the health care context, also the effects of the observed practice meanings and finally the care giver way of acting and relating. The carers build medical encounter and distance relationships. This is presented as the "world-body" concept. As part of the new care exercises and the nurse teaching evolution, the health care organizations are faced with strategic questionings. In order to answer those interrogations, the central concept of "communicatingcare" is developed. This leads to define the care act as a meaningful and complex interaction system. This concept tries to formalize mediation areas which are specific to the building of a relevant and coherent caring professionalism as regards with looking at the new challenges in nurse care teaching.
\end{abstract}

Keywords: care, training, body, mediation, values, identity, activity. 